\title{
Successful Non Contrast Percutaneous Coronary Intervention for Patient with Unstable Angina and Prior Anaphylactic Reaction to Iodinated Contrast Medium
}

\author{
Mamoru Sakakibara and Hiroyuki Tsutsui
}

\begin{abstract}
A 53-year-old man, who had a history of angina treated by percutaneous coronary intervention without allergic reaction to contrast and coronary bypass surgery, was hospitalized due to epigastralgia and tarry stool. During an enhanced computed tomography for the evaluation of abdominal diseases, he became hypotensive and had chest pain. To diagnose acute coronary syndrome, coronary angiography (CAG) was performed after the intravenous administration of hydrocortisone. He became hypotensive again during the CAG, which revealed significant coronary artery stenosis in the right coronary artery. Despite the intensive medical treatment, he had angina even while walking in the ward. By using an intravascular ultrasound for coronary stent implantation and the second wire as a marker for the stent implantation, we performed coronary interventional procedures successfully for this patient without the use of iodinated contrast media.
\end{abstract}

Key words: anaphylaxis, coronary intervention, iodinated contrast media, intra vascular ultrasound (IVUS)

(Inter Med 48: 1753-1757, 2009)

(DOI: 10.2169/internalmedicine.48.2437)

\section{Introduction}

More than 70 million diagnostic radiographic examinations using iodinated contrast media (ICM) are performed worldwide each year (1). Anaphylactoid reactions to ICM are one of the common complications during these examinations. Clinical manifestations of systemic anaphylaxis include various combinations of flushing, urticaria laryngeal edema, and hypotension. The incidence of a severe reaction such as shock is extremely low (less than one death per 100,000 patients) (2-7). However, ICM-related adverse reactions should be prevented and appropriately treated when they do occur. This is our first report of successful percutaneous coronary intervention (PCI) without the use of ICM in a patient with unstable angina and prior systemic anaphylaxis to ICM.

\section{Case Report}

A 53-year-old man, who had a history of hypertension and angina pectoris, received coronary angiography (CAG) including percutaneous coronary intervention (PCI) with ICM (Iomeron $350^{\mathrm{TM}}$, Eisai, Tokyo, Japan) 5 times and a coronary artery bypass graft (CABG) surgery. He had never experienced an ICM allergy before. PCI with bare metal stent (BMS) was performed for the 75\% stenosis of the mid left circumflex artery (Lcx) on May, 2004. However, the stenotic lesions of all three coronary arteries progressed afterwards. Follow-up CAG revealed chronic total occlusion (CTO) in the proximal left anterior descending artery (LAD), in-stent $75 \%$ restenosis in the proximal Lcx and $50 \%$ stenosis in the mid right coronary artery (RCA) in November, 2004. Therefore, he underwent CABG in December, 2004, receiving a left internal mammary artery (LIMA) to Lcx and right internal mammary artery (RITA) to LAD in another hospital. Follow-up CAG revealed that RITA to LAD was patent, but the LIMA graft was totally occluded. We successfully performed PCI for in-stent $75 \%$ restenosis in the proximal Lcx by using cutting balloon in July, 2005. Afterwards he had no angina attacks.

$\mathrm{He}$ was hospitalized due to epigastralgia and tarry stool in

Department of Cardiovascular Medicine, Hokkaido University Graduate School of Medicine, Sapporo

Received for publication May 4, 2009; Accepted for publication June 29, 2009

Correspondence to Dr. Mamoru Sakakibara, mike.s@ninus.ocn.ne.jp 

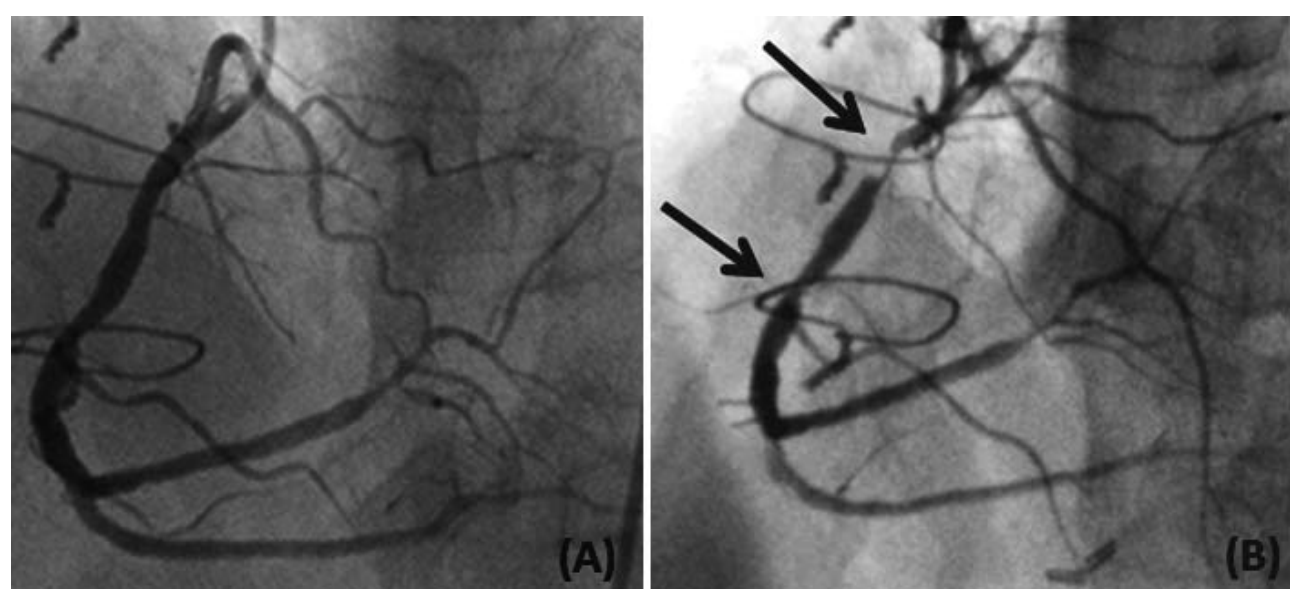

Figure 1. Previous coronary angiographic findings of the right coronary artery (A) and those obtained from the hospitalization for epigastralgia and tarry stool (B). Severe stenotic lesions were demonstrated at the proximal and the middle portion of right coronary artery, which had obviously progressed compared to the lesions on the earlier angiograms (arrows denoted significant stenosis).

May, 2007.

The hemoglobin value was $11.0 \mathrm{mg} / \mathrm{dL}$ on admission, and was slightly decreased from the previous value $(12.6 \mathrm{mg} /$ dL). During computed tomography with $100 \mathrm{~mL}$ of ICM [Optiray $320^{\mathrm{TM}}$, (Ioversol), Tyco Healthcare, Tokyo, Japan] for abdominal evaluation, his blood pressure dropped from $110 \mathrm{mmHg}$ to $60 \mathrm{mmHg}$ suddenly with chest pain, dyspnea, and flushing lasting approximately 10 minutes. We diagnosed him as shock due to systemic anaphylaxis to ICM, however, could not completely exclude the contribution of coronary ischemic event. We thus performed CAG using ICM which we safely used for him before (Iomeron $350^{\mathrm{TM}}$, Eisai, Tokyo, Japan) after an intravenous administration of hydrocortisone $500 \mathrm{mg}$. However, his blood pressure decreased to $50 \mathrm{mmHg}$ again for 10 minutes at the end of the CAG. We immediately administered epinephrine $(1: 1,000)$ $0.5 \mathrm{mg}$ intramuscularly, diphenhydramine $50 \mathrm{mg}$ and hydrocortisone $500 \mathrm{mg}$ intravenously, and subsequently infused $1,000 \mathrm{~mL}$ of lactated Ringer solution rapidly. After recovery, we completed CAG using $35 \mathrm{~mL}$ of ICM and found significant $75 \%$ and $90 \%$ stenosis at the proximal and middle regions of the right coronary artery (RCA), but no significant stenosis at the left anterior descending (LAD) and left circumflex (Lcx) coronary artery (Fig. 1).

Despite the intensive medical treatment, he had angina attacks even while walking in the ward. PCI was performed using intravascular ultrasonography (IVUS) without using ICM. To prevent anaphylaxis, we administered him prednisone $20 \mathrm{mg}$ orally before 13 hours, 7 hours, and 1 hour and diphenhydramine $50 \mathrm{mg}$ parenterally 1 hour before the procedures as premedication. During the PCI procedure, a $6 \mathrm{Fr}$ JR 4.0 guiding catheter (Cordis, NJ, USA) was positioned at the ostium of the RCA. A 0.014-inch Rinato guidewire (Termo, Tokyo, Japan) was advanced into the distal RCA and a 0.014-inch Route guidewire (Asahi Intec, Aichi, Japan) was also put into the right ventricular branch (RV) as a marker for stent implantation (Fig. 2). IVUS imaging using $40 \mathrm{MHz}$ Atlantis IVUS catheter (Boston Scientific, Natick, MA, USA) demonstrated moderate calcification in the proximal and the mid RCA. Pull-back speed was set at $0.5 \mathrm{~mm} /$ sec to measure the accurate length of the stenotic lesion by IVUS system. Pre-dilation before stenting was performed using a $2.75 \times 20 \mathrm{~mm}$ Splinter balloon catheter (Medtronic, MN, USA). By using IVUS guide for stenting and the second wire as a marker for the implantation of coronary stent, we carefully implanted a $3.0 \times 32 \mathrm{~mm}$ stent (Taxus Express ${ }^{\mathrm{TM}}$, Boston Scientific, Natick, MA, USA) and the final IVUS imaging showed successful results (Fig. 3). We found by gastric endoscopy that the cause of tarry stool was a small gastric ulcer. Fortunately, it was at the healing stage and we could choose a drug eluting stent.

\section{Discussion}

Under the guide by IVUS images, PCI could be successfully performed without using ICM in a patient with unstable angina and prior anaphylactic shock to ICM. We employed 3 therapeutic strategies to perform PCI successfully and safely for this patient. First, we used previous CAG findings for the basis of the information about the lesion of location and length for performing PCI, which enabled us to easily confirm the relationship between the stenotic lesion and the side branch. We also could identify the stenotic lesion of the mid RCA located at the right ventricular branch (RVB). Second, we advanced one wire into the distal RCA and another wire into the RV branch, which was located around the second stenosis at the mid portion of RCA, as a marker for the implantation of coronary stent. Third, we used IVUS imaging, which allowed not only measurement of the diameter of the vessel and view of the characteristics of the lesion such as calcification and attenuated plaque, but also facilitated careful determination of the stent length and 

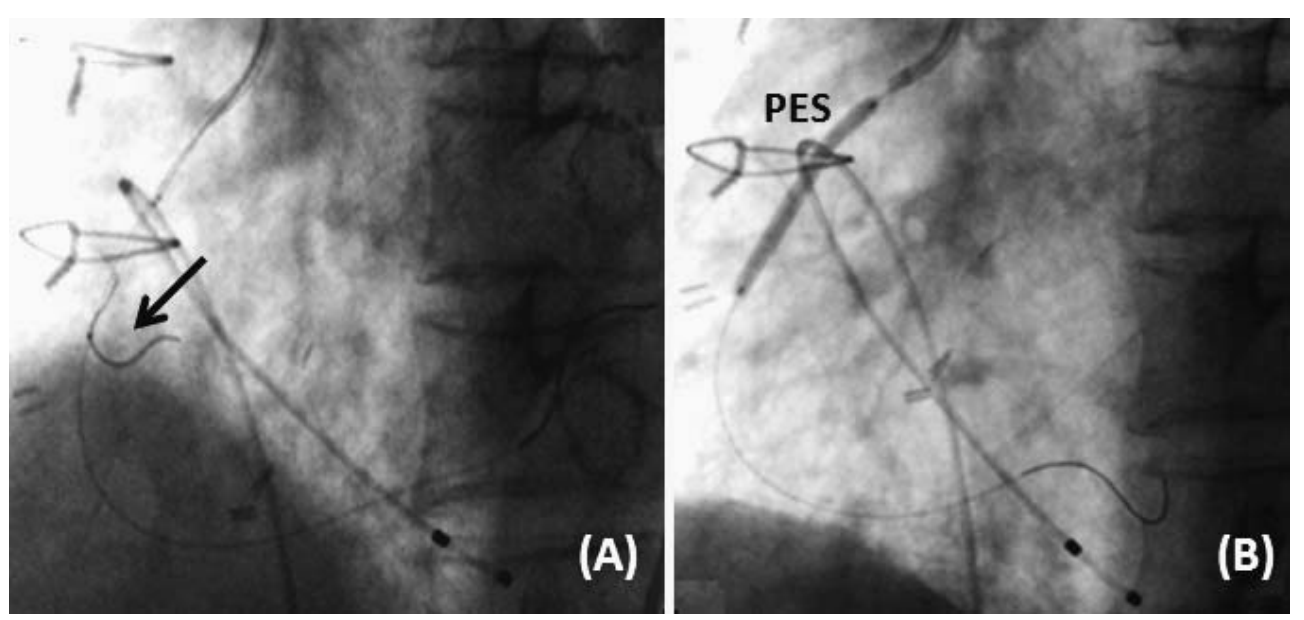

Figure 2. (A) The second wire was advanced into the right ventricular branch (arrows), which was located around the second stenosis at the mid portion of RCA. This wire was used as one of the markers for stenting. (B) A Taxus Express ${ }^{\mathrm{TM}}$ Stent (PES) was implanted under the guidance of the IVUS and the second wire. The second wire was removed carefully just before the stent dilation.
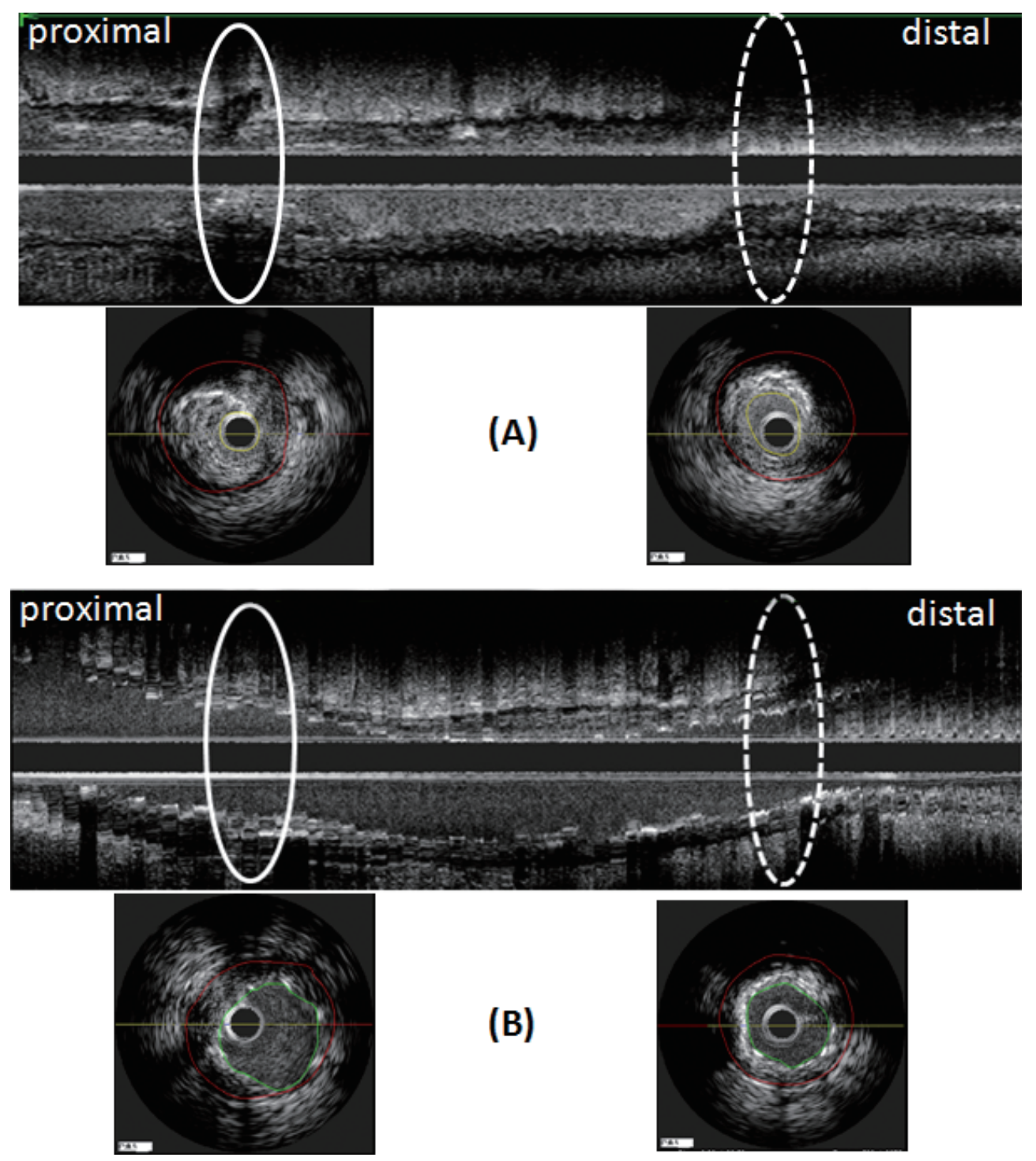

Figure 3. Intracoronary ultrasound (IVUS) imaging: (Upper: longitudinal view, Lower: axial view). (A) Pre-procedural IVUS revealed two stenotic lesions, located at the proximal (circle of a solid line) and the middle (circle of a dotted line) portion of right coronary artery. (B) Post-procedural IVUS image demonstrated the optimal results. 
the location. We were able to readily place the stent by marking the initiation and termination of the lesion by a cineradiography using a binary marker at the tip of IVUS. Moreover, after implanting the coronary stent, we could check for the possible presence of injury or hematoma at the edge of the stent. However, IVUS-guided PCI without the use of ICM may cause complications including distal wireinjury or slow / no flow phenomenon due to distal embolism, which may occur with the use of the polymer jacket wire in particular. Moreover, the slow/no flow phenomenon can be diagnosed only by the symptoms of the patient or based on the findings of electrocardiograms.

Patients who have experienced an anaphylactoid reaction to ICM are at greatest risk to suffer another episode as seen in the present patient. In such patients with recurrent systemic anaphylaxis, prophylactic use of histamine 1 receptor antihistamine is beneficial $(8,9)$. Prednisone orally or intravenous (may also reduce the risk of recurrent anaphylaxis. However, it is necessary to understand that prednisone is not effective if given less than 6 hours before ICM due to its slow acting property. Furthermore, some controlled clinical trials have already revealed the combination of prednisone and histamine 1 receptor antihistamine to be the most beneficial in preventing anaphylactoid reactions to $\operatorname{ICM}(8,9)$. In addition, ephedrine, which is used as a bronchodilator of the $\beta 2$ receptor stimulant, has been sometimes added in a few clinical trials (8). However, the addition of ephedrine to decrease the prevalence of adverse reactions to ICM has not gained wide acceptance. Ephedrine is contraindicated for uncontrollable hypertension and unstable angina because it also releases norepinephrine from sympathetic nerve endings, which makes $\alpha$ - and $\beta 1$-adrenergic stimulants activate (10). Based on these findings, we did not administer ephedrine to our patient.

The availability of newer low- and iso-osmolar nonionic contrast agents adds a further margin of safety since the rate of severe cross-reaction with prior reaction to an ICM is also high. For this indication, the true nonionic agents are preferable. We did not use the "generic" contrast in this patient which may contain ingredients which cause anaphylactoid reactions. Even though this patient received the same original nonionic contrast agents which we had used many times before, he had anaphyractoid shock during the CAG.
In patients with a severe prior allergic reaction to ICM, the use of gadolinium chelates may be considered in place of ICM. In fact, several reports have demonstrated that gadolinium chelates could be an alternative contrast media during PCI in particular patients with prior allergy to ICM (11, 12). The prevalence of anaphylactoid reactions to gadolinium chelates is reported to be extremely low at 0.0002 $0.001 \%$. However, the risk of adverse reactions may increase by 3 to 4 fold in patients with a history of anaphylactoid reaction to $\operatorname{ICM}(12,13)$. In addition, another limitation of gadolinium is its low radiopacity. Moreover, gadolinium chelates have adverse effects including nephritic systemic fibrosis especially for patients with renal dysfunction. Therefore, we performed the PCI under the guide of IVUS along with anphylactoid prophylaxis beforehand. In the case that some complications, such as a slow flow/ no flow, may occur with chest pain or the disturbance of vital signs, we had planned to perform coronary angiography immediately with the use of ICM to confirm the diagnosis. In the case of an emergency, we had the approach to prevent a slow flow/ no flow phenomenon including the use of intracoronary infusion of verapamil, diltiazem, adenosine, or nitroprusside at the time of the procedure $(14,15)$. Nevertheless, it is important to emphasize again that the prevention of the contrast allergy in advance is extremely cardinal.

Oxygen supplementation, intravenous administration of physiological fluids and intramuscular injection of 0.3 to 0.5 mg epinephrine $(1: 1,000)$ should be considered in the first line of management of acute anaphylaxis.

In conclusion, PCI could be successfully and safely performed for a patient with unstable angina and prior anaphylactic shock to ICM under IVUS guide and the second wire as a marker for coronary stenting.

\section{Acknowledgement}

We thank Dr. Yosito Sakata and Yasufumi Hayama in Ikegami General Hospital, Dr. Masakazu Nagaoka and Dr. Naoko Tsumuraya in the Department of Cardiology, Tokai University School of Medicine, and Dr. Shintaro Kinugawa, Dr. Nozomu Kawashima and Dr. Takamitsu Souma of Department of Cardiovascular Medicine, Hokkaido University Graduate School of Medicine for encouragement and critical reading of the manuscript.

\section{References}

1. Christiansen C. X-ray contrast media--an overview. Toxicology 209: 185-187, 2005.

2. Katayama H, Yamaguchi K, Kozuka T, Takashima T, Seez P, Matsuura $\mathrm{K}$. Adverse reactions to ionic and nonionic contrast media. A report from the Japanese Committee on the Safety of Contrast Media. Radiology 175: 621-628, 1990.

3. Shehadi WH, Toniolo G. Adverse reactions to contrast media: a report from the Committee on Safety of Contrast Media of the International Society of Radiology. Radiology 137: 299-302, 1980.

4. Cochran ST, Bomyea K, Sayre JW. Trends in adverse events after IV administration of contrast media. AJR Am J Roentgenol 176:
1385-1388, 2001.

5. Greenberger PA, Patterson R. The prevention of immediate generalized reactions to radiocontrast media in high-risk patients. J Allergy Clin Immunol 87: 867-872, 1991.

6. Bettmann MA, Heeren T, Greenfield A, Goudey C. Adverse events with radiographic contrast agents: results of the SCVIR Contrast Agent Registry. Radiology 203: 611-620, 1997.

7. Caro JJ, Trindade E, McGregor M. The risks of death and of severe nonfatal reactions with high- vs low-osmolality contrast media: a meta-analysis. AJR Am J Roentgenol 156: 825, 1991.

8. Greenberger PA, Patterson R, Radin RC. Two pretreatment regi- 
mens for high-risk patients receiving radiographic contrast media. J Allergy Clin Immunol 74: 540-543, 1984.

9. Tramer MR, von Elm E, Loubeyre P, Hauser C. Pharmacological prevention of serious anaphylactic reactions due to iodinated contrast media: systematic review. BMJ 333: 675, 2006.

10. Morcos SK, Thomsen HS, Webb JA. Contrast Media Safety Committee of the European Society of Urogenital Radiology. Prevention of generalized reactions to contrast media: a consensus report and guidelines. Eur Radiol 11: 1720-1728, 2001.

11. Furuichi S, Yasuda S, Arita $Y$, et al. Gadopentetate dimeglumine as a potential alternative contrast medium during percutaneous coronary intervention: a case report. Circ J 68: 972-973, 2004.

12. Spinosa DJ, Kaufmann JA, Hartwell GD. Gadolinium chelates in angiography and interventional radiology: a useful alternative to iodinated contrast media for angiography. Radiology 223: 319327, 2002.

13. Murphy JM, O'Hare NJ, Smiddy P, Molloy MP. Gadorpentetate dimegumine as a contrast agent in peripheral angioplasty: A case report. Acta Radiol 39: 576-578, 1998.

14. Marzilli M, Orsini E, Marraccini $P$, Testa R. Beneficial effects of intracoronary adenosine as an adjunct to primary angioplasty in acute myocardial infarction. Circulation 101: 2154-2159, 2000.

15. Taniyama $\mathrm{Y}$, Ito $\mathrm{H}$, Iwakura $\mathrm{K}$, et al. Beneficial effect of intracoronary verapamil on microvascular and myocardial salvage in patients with acute myocardial infarction. J Am Coll Cardiol 30: 1193-1199, 1997.

(C) 2009 The Japanese Society of Internal Medicine http://www.naika.or.jp/imindex.html 